\title{
Relación entre uso problemático de internet y calidad de sueño durante la pandemia de COVID-19
}

\author{
Samuel Olegario Iñiguez Jiménez \\ ORCID: https://orcid.org/0000-0002-4722-7611 \\ iniguezjimenez@gmail.com \\ Pontificia Universidad Católica del Ecuador \\ Quito, Ecuador \\ Stephanie Marie Cruz Pierard \\ ORCID: https://orcid.org/0000-0003-4100-5898 \\ smcp0893@gmail.com \\ Pontificia Universidad Católica del Ecuador \\ Quito, Ecuador
}

\author{
Isaac Josué Iñiguez Jiménez \\ ORCID: https://orcid.org/0000-0002-4136-1959 \\ isaaciniguez98@gmail.com \\ Universidad Politécnica Salesiana \\ Quito, Ecuador \\ Alicia Carolina Iñiguez Jiménez \\ ORCID: https://orcid.org/0000-0002-9350-3437 \\ carolinainiguezj@gmail.com \\ Pontificia Universidad Católica del Ecuador \\ Quito, Ecuador
}

Recibido (26/04/21), Aceptado (14/05/21)

Resumen: Se analizó la relación entre el uso problemático de internet y la calidad de sueño. La metodología fue no experimental, transversal y correlacional. La muestra fue no probabilística por conveniencia. Participaron 118 estudiantes de pregrado y postgrado de universidades e institutos superiores de la ciudad de Quito en Ecuador. Los instrumentos fueron el Cuestionario de Experiencias Relacionadas con Internet (CERI) y el Cuestionario de Pittsburg de Calidad de Sueño (PSQI). Se evaluó la asociación entre las variables mediante el coeficiente de correlación de Pearson y un valor $\mathrm{p}<0,05$ durante la emergencia sanitaria por COVID-19. Los resultados muestran que los participantes en $51 \%$ tienen problemas ocasionales con el uso de internet y el $47 \%$ tienen problemas de sueño que además merecen atención y tratamiento médico. La asociación entre la variable independiente y la variable dependiente fue $r=0,195$ y $p=0,034$. En conclusión, existe una relación significativa moderada entre el uso problemático de internet y la calidad de sueño.

Palabras Clave: Calidad de sueño, estudiantes universitarios, salud mental, uso de internet.

\section{Relationship between problematic internet use and sleep quality during the COVID-19 pandemic}

\begin{abstract}
The relationship between problematic internet use and sleep quality was analyzed. The methodology was non-experimental, cross-sectional, and correlational. The sample was non-probabilistic for convenience. 118 undergraduate and graduate students from universities and higher institutes from the city of Quito in Ecuador participated. The instruments were the Internet-Related Experiences Questionnaire (CERI) and the Pittsburg Sleep Quality Questionnaire (PSQI). The association between the variables was evaluated using Pearson's correlation coefficient and a $p$-value $<0,05$ during the health emergency due to COVID-19. The results show that $51 \%$ of the participants have occasional problems with internet use and $47 \%$ have sleep problems that also deserve medical attention and treatment. The association between the independent variable and the dependent variable was $r=0,195$ and $p=0,034$. In conclusion, there is a moderately significantrelationship between problematic internetuse and sleep quality.
\end{abstract}

Keywords:Internet use, mental health, sleep quality, university students. 


\section{Introducción}

En los últimos años el uso de internet ha sido ampliamente difundido a nivel mundial [1]; siendo una herramienta tecnológica utilizada en todas las edades para diversos fines, no solo relacionados con la comunicación o esparcimiento, si no también indispensable tanto en educación, investigación y salud [2].

Por otra parte, la calidad de sueño es un elemento relevante para la vida, el cuerpo humano funciona con un ritmo circadiano [3], que es un proceso biológico que tiene lugar durante un período de 24 horas. Este proceso se basa principalmente en la salida y la caída del sol. Una parte del cerebro llamado núcleo supraquiasmático utiliza señales ambientales como la luz para saber cuándo es el momento de iniciar el sueño. Además, este núcleo es muy sensible incluso a pequeños cambios en el entorno [3], [4].

Los dispositivos electrónicos con pantalla emiten luz [4]. Esta radiación electromagnética tiene una longitud de onda corta que interfiere con el ritmo circadiano al retrasar la producción de melatonina por la noche [4]. La interrupción en la liberación de melatonina puede provocar insomnio [5]. Por lo que alterar el ciclo vigilia-sueño tiene consecuencias negativas a nivel biológico, psicológico y social.

En este aspecto, el número de horas de trabajo, actividades académicas y lúdicas se ha visto incrementado en un 12 $\%$ desde los años sesenta hasta los dos mil [6]. El periodo de tiempo de uso de internet empezó a multiplicarse mundialmente, asimismo las horas de sueño se han reducido de 9 horas en los años 50 a 7 horas en la población activa actual [6].

Es preciso señalar que las personas con uso problemático de internet experimentan ciertos síntomas de irritabilidad, ansiedad y anomalías emocionales [7], al mismo tiempo presentan conductas alarmantes como el descuido de sus quehaceres diarios por permanecer conectados a la red, sacrificando horas de descanso nocturno [8].

Al considerar las razones expuestas previamente se evidencia la importancia de realizar la presente investigación cuyo objeto consistió en analizar la relación entre el uso problemático de internet y la calidad de sueño, especialmente en Ecuador, debido a la escasez de estudios que reflejen la relación de estas variables en estudiantes universitarios. Se busca dar respuesta a la siguiente pregunta de investigación: ¿Cuál es la relación entre el uso problemático de internet y la calidad de sueño?

\section{Desarrollo}

El internet se define como una red informática mundial grande, cambiante, diversa y descentralizada [9], que nos permite acceder a variadas fuentes de información, ocio, actividades para el desarrollo personal, académico y profesional, pero también trae consigo innegables riesgos asociados como el aislamiento, la pérdida de privacidad y el contacto con gente desconocida [10]. Además, en ocasiones su utilización se puede tornar excesiva y a su vez problemática, causando alteraciones a nivel físico y psicológico [2].

El concepto uso problemático de internet, describe la in- capacidad de una persona para controlar su utilización, generando un malestar psicológico y una afectación funcional; en cuanto a la literatura psicológica, lo ha definido como un uso patológico o adicción basada en los criterios que caracterizan al juego patológico o consumo de sustancias según el Manual Diagnóstico y Estadístico de Trastornos Mentales en su cuarta edición (DSM-IV), originando el término desorden de adicción al internet (IAD) [8].

En este contexto, hay algunos estudios que han demostrado que el uso problemático del internet puede influir en varios aspectos del aprendizaje y la salud de las personas, como es el caso de una investigación en la cual concluyeron que el uso problemático del internet se asoció significativamente con la presencia de migrañas, descanso insuficiente, dolor lumbar, sobrepeso, así como con alteraciones psicológicas (depresión, riesgo de trastornos alimentarios y mentales), discriminación y problemas familiares [11]. De igual manera, en un estudio realizado en 5 universidades de Colombia se determinó que el 77,3\% de los participantes presentaron adicción al internet, la misma que mostró una relación estadísticamente significativa con depresión, promedio de notas, hiperactividad, inatención, somnolencia, entre otros [12].

Asimismo, existen varias investigaciones que analizan la calidad de sueño en universitarios relacionada con algunos factores entre ellos la adicción al internet, por ejemplo, la realizada en la Universidad Católica Santo Toribio de Mogrovejo en Perú en la que el $85 \%$ de estudiantes presentaron una calidad deficiente de sueño asociada a la carga académica que consecuentemente conlleva el uso de internet para realizar trabajos e investigaciones [13]. Del mismo modo, en un estudio llevado a cabo en estudiantes de múltiples universidades de China [14] se determinó que existe una asociación significativa entre el uso problemático de internet con la disminución de la calidad del sueño $(r=0,32, p<0,001)$.

En Ecuador existen pocas investigaciones sobre este tema, más que todo enfocadas en poblaciones jóvenes, sin embargo, se encontró un estudio [15] sobre la calidad de sueño y los factores que se asocian en internos rotativos de medicina pertenecientes a la Universidad de Cuenca, en el que se concluyó según el Índice de Pittsburgh, que el 99.3 \% de las personas estudiadas, presentaban mala calidad de sueño, sin encontrarse asociaciones significativas con consumo de cafeína, tabaco o características generales como edad, género o tipo de rotación.

Por otro lado, es importante recalcar que la pandemia de COVID-19 ha concebido que el uso del internet se vea incrementado en la población y a su vez ha modificado sus horarios y calidad de sueño. Según una publicación [16] el uso de internet en los hogares ecuatorianos ascendió hasta 63 $\%$ durante el confinamiento, pudiendo deberse a que 425000 empleados de empresas del sector público y privado fueron obligados a trabajar en sus casas por el distanciamiento social, al igual que 4 millones de estudiantes a recibir clases online. Del mismo modo, en una investigación realizada en universitarios de diversos países [17] se observó un aumento del uso de internet, especialmente en cuanto al acceso a 
redes sociales y servicios de transmisión, adicionalmente se determinó que los participantes con alta puntuación en uso compulsivo de internet y redes sociales obtuvieron también valores altos en mala calidad del sueño, aversión, soledad, depresión y ansiedad afín a la pandemia, por ende se deduce que sin importar el país, el confinamiento por COVID-19 ha mostrado un impacto notable en el uso de internet y el bienestar psicosocial.

\section{Metodología}

El presente estudio se encuentra enmarcado en un diseño no experimental, transversal, correlacional. El tipo de muestreo fue no probabilístico por conveniencia y la población se conformó por una muestra de 118 personas que cumplieron con los siguientes criterios:

-Estudiantes universitarios de pregrado y postgrado.

-En edades comprendidas entre 18 y 60 años de edad.

-Pertenecientes al Distrito Metropolitano de Quito, Ecuador.

La recolección de datos se realizó mediante la aplicación de encuestas virtuales a través de la plataforma Google Forms ${ }^{\circledR}$ durante la pandemia por COVID-19 en los meses de octubre, noviembre y diciembre del 2020. El instrumento estuvo conformado por los siguientes cuestionarios:

- Cuestionario de Experiencias Relacionadas con Internet (CERI). Este cuestionario mantiene una fiabilidad según el Alfa de Cronbach del 0,77 [18].

- Cuestionario de Pittsburg de Calidad de Sueño (PSQI).
Este instrumento presenta una fiabilidad según el Alfa de Cronbach del 0,83 [19].

En cuanto al procedimiento, se aplicó encuestas virtuales, previo al beneplácito del consentimiento informado, se estableció como requisito tener todo el documento cumplimentado para poder enviarlo a través de la red, así se evitó perdida de información.

La tabulación de datos se realizó en el programa Microsoft Excel ${ }^{\circledR}$ versión 2101, el análisis de los mismos se efectuó mediante el programa SPSS ${ }^{\circledR} 18$. En primera instancia se caracterizó a los participantes mediante la edad, sexo y ocupación. Posteriormente, para analizar la relación entre la variable independiente (uso problemático de internet) y la variable dependiente (calidad de sueño) se utilizó el coeficiente de correlación de Pearson con el $5 \%$ de nivel de significancia.

\section{Resultados}

El mundo está en constante desarrollo y en la actualidad se ha visto influenciado por diversas tecnologías de la información que tienen como objetivo principal hacer que la vida humana sea más fácil y llevadera, un claro ejemplo es el uso del internet, siendo el instrumento más usado a nivel mundial para diversos fines, especialmente por los más jóvenes [20]. Sin embargo, a pesar de los varios aspectos positivos y utilidades que presenta, también puede conllevar riesgos si su uso se vuelve patológico o adictivo, lo cual en muchas ocasiones se subestima [21].

Tabla 1. Caracterización de la población

\begin{tabular}{|c|c|c|c|}
\hline Variable & & Frecuencia & Porcentaje $(\%)$ \\
\hline \multicolumn{4}{|c|}{ Género } \\
\hline Femenino & 82 & & 69,49 \\
\hline Masculino & 36 & & 30,51 \\
\hline \multicolumn{4}{|c|}{ Ocupación } \\
\hline Estudia & 46 & & 38,98 \\
\hline Trabaja & 45 & & 38,14 \\
\hline Estudia y Trabaia & 27 & & 22,88 \\
\hline \multicolumn{4}{|c|}{ Edad (años) } \\
\hline Media & Mediana & Moda & Desviación típica \\
\hline 28 & 25 & 23 & 9,52 \\
\hline
\end{tabular}

En el presente estudio (tabla 1) la edad más frecuente es igual a la estimada en estudiantes universitarios del Ecuador, 23 años [22]. Conforme con lo mencionado anteriormente en varias investigaciones concuerdan que los universitarios son una de las poblaciones más afectadas por el uso problemático del internet, siendo más prevalente entre los 16-26 años [12], [23]. 


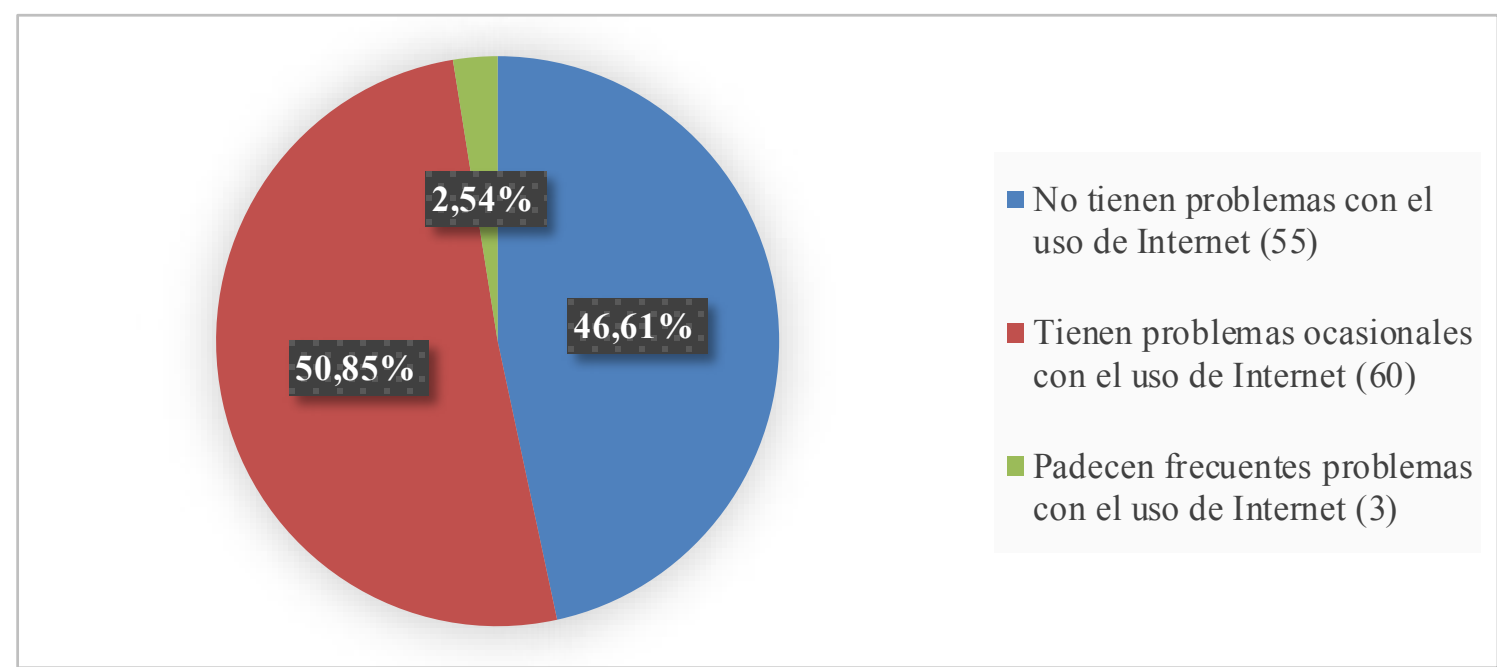

Fig. 1. Distribución del uso problemático del internet

Según los baremos del CERI (figura 1) 5 de cada 10 estudiantes tienen problemas ocasionales de uso de internet, lo cual coincide con los resultados obtenidos en una investigación realizada en la Universidad de Cádiz [24] en la que se determinó que el 44,5\% de los universitarios presentaron problemas ocasionales y el 5,7 \% problemas frecuentes, evidenciando que más de la mitad de la población estudiada manifiesta un uso problemático de internet. Según varios autores [25] estas conductas tienden a darse reiteradamente en los participantes más jóvenes y las principales actividades realizadas en internet son chatear, acceder a redes sociales, juegos y blogs.

Tabla 2. Media, mediana y moda del uso problemático del internet

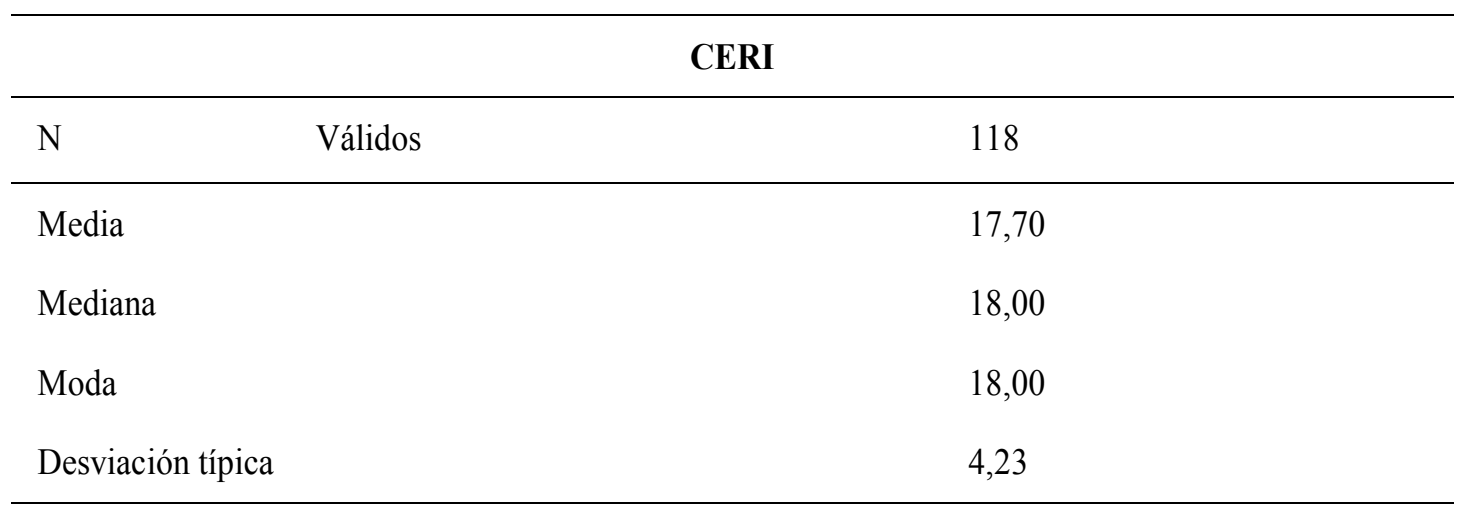

El puntaje del CERI (tabla 2) indica que alrededor del 50 $\%$ de la población reporta un elevado uso problemático de internet $(>18)$, de igual manera el valor más frecuente corresponde a personas con problemas ocasionales con el uso de internet. Acotando, el uso exagerado de las Tecnologías de Información y Comunicación (TICs), principalmente en jóvenes, genera una baja autoestima y mayor dificultad de socializar [26]. Esta evidencia propone cambiar la forma en que los estudiantes interactúan con la tecnología antes de acostarse. 


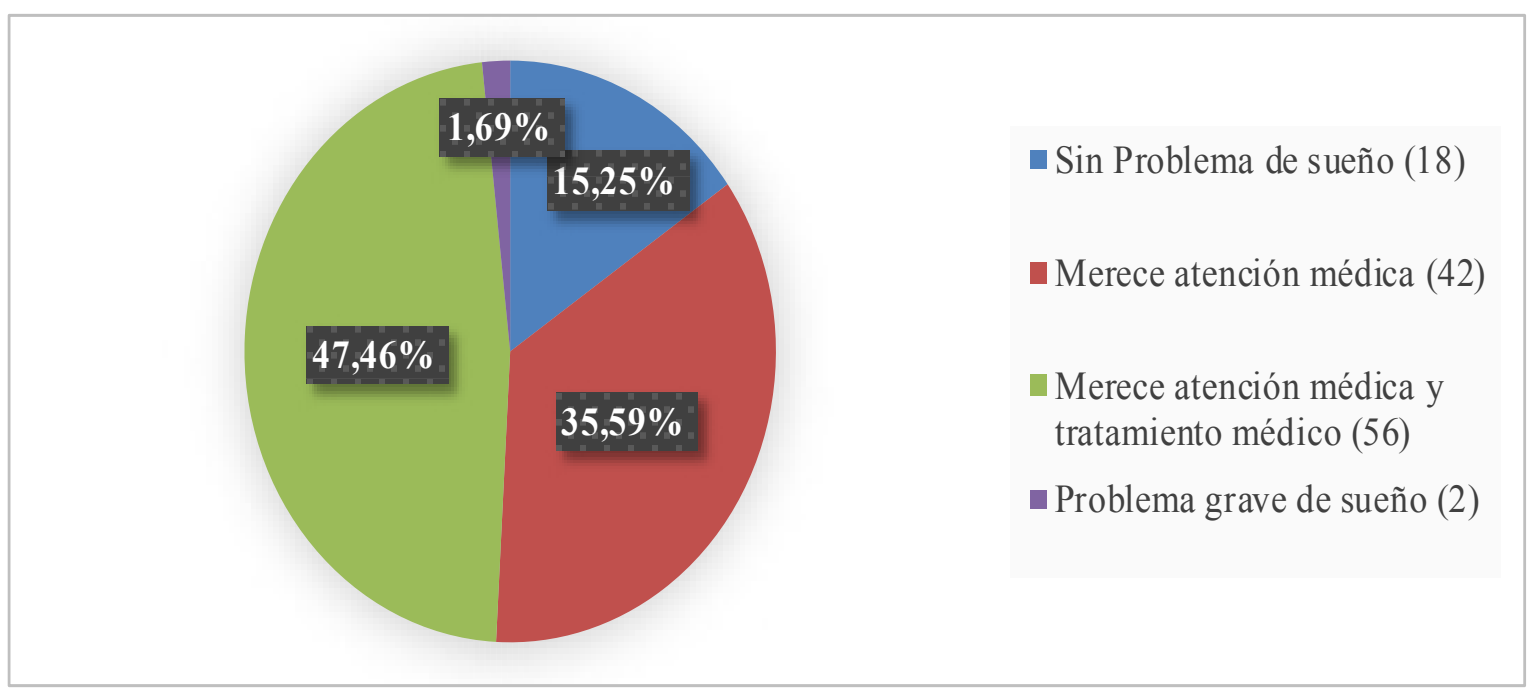

Fig. 2. Distribución de la calidad de sueño

Por otro lado, en cuanto a la calidad de sueño la mayoría de estudiantes universitarios muestran una deficiencia de la misma, la cual afecta su salud y conduce a la necesidad de una evaluación y tratamiento médico [15], [27]. La figura 2 indica que el 84,75\% de los participantes de esta investigación presentan problemas de sueño, siendo esencial conside- rar la necesidad de atención médica, tal como se evidencia en un estudio desarrollado en Colombia [28] en el cual mostraron resultados similares $(77.1 \%)$ y recalcan que la pobre calidad de sueño requiere una mayor educación en relación con la higiene del mismo.

Tabla 3. Media, mediana y moda de la calidad de sueño

\begin{tabular}{lll}
\hline & PSQI \\
\hline $\mathrm{N}$ & Válidos & 118 \\
\hline Media & 7,4492 \\
Mediana & 7,0000 \\
Moda & 8,00 \\
Desviación típica & 2,92527 \\
\hline
\end{tabular}

La tabla 3 indica que, con un puntaje de 7,00 según el PSQI, el $50 \%$ de la muestra reporta una calidad baja de sueño. Tanto el promedio como el valor con mayor frecuencia (8), corresponde a personas con problemas de sueño que merecen atención y tratamiento médico. Los factores del estilo de vida afectan con mayor fuerza la calidad del sueño entre los estudiantes universitarios, seguidos de los factores mentales, sociales y físicos [29]. Por ende, se debe tomar medidas para promover una buena higiene del sueño para que los estudiantes se sientan más saludables, alertas y descansados. 


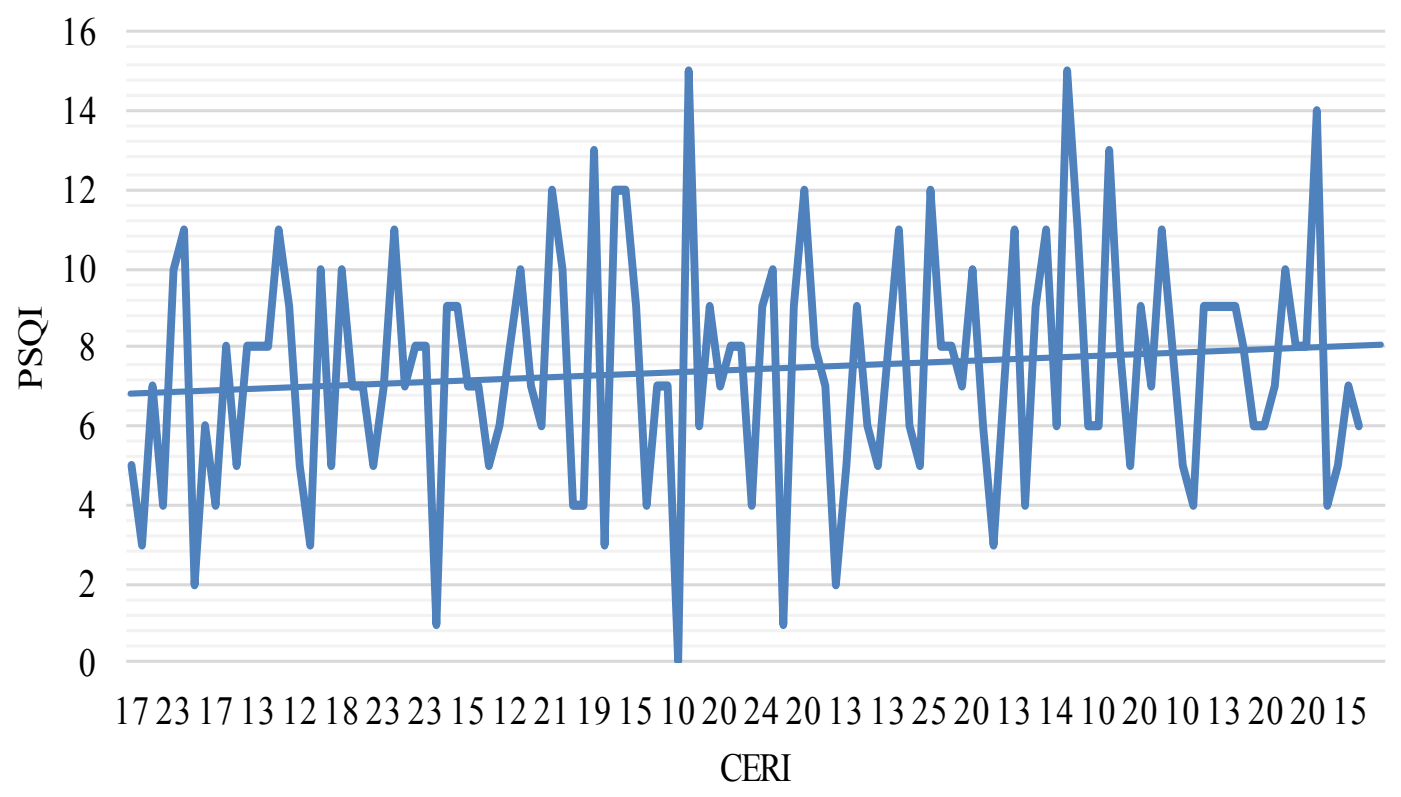

Fig. 3. Relación entre el uso problemático del internet y la calidad de sueño

Tabla 4. Relación entre el uso problemático del internet y la calidad de sueño

\begin{tabular}{llrr}
\hline \multicolumn{1}{c}{ Correlaciones } & & \\
\hline & & $\begin{array}{c}\text { Ceri (Uso } \\
\text { Problemático De } \\
\text { Internet) }\end{array}$ & $\begin{array}{c}\text { Pittsburgh } \\
\text { (Calidad De } \\
\text { Sueño) }\end{array}$ \\
\hline Ceri (Uso Problemático De & Correlación de Pearson & 1 & $0,195^{*}$ \\
Internet) & Sig. (bilateral) & 118 & 0,034 \\
& $\mathrm{~N}$ & $0,195^{*}$ & 118 \\
\hline Pittsburgh (Calidad De & Correlación de Pearson & 0,034 & 1 \\
Sueño) & Sig. (bilateral) & 118 & 118 \\
& $\mathrm{~N}$ & & \\
\hline *. La correlación es significante al nivel 0.05 (bilateral). & & \\
\hline
\end{tabular}

Con los datos expuestos en la figura 3 se realizó una correlación entre la variable independiente (uso problemático de internet) y la dependiente (calidad de sueño). De esta manera la tabla 4 presentó suficiente evidencia empírica $(r=0,195$ y $\mathrm{p}=0,034)$ para concluir que existe una relación estadísticamente significativa moderada entre el uso problemático del internet y la calidad de sueño. Algunos autores corroboran que existe esta asociación [14], entre ellos se destaca un estudio [2] realizado utilizando una metodología diferente (razones de prevalencias crudas RPc y ajustadas RPa) en el que se obtuvo como resultado que el uso problemático de internet se relaciona con una mala calidad de sueño (RPa: 1,51; IC 95 $\%: 1,01-2,23)$. De igual manera, en una investigación llevada a cabo en una universidad mexicana [30] se determinó que los estudiantes que pasaban más tiempo conectados a internet se iban a dormir más tarde afectando su calidad de sueño $(\mathrm{r}=$ $0,301, p=0,003)$.

Es importante mencionar que el uso de internet aumentó considerablemente durante la pandemia de COVID-19, lo que pudo haber generado o empeorado estos problemas en la población y a su vez afectados su calidad de sueño. Según un informe realizado por el Ministerio de Sanidad de España [31] sobre COVID-19, consumo de sustancias psicoactivas y adicciones, el 68,9\% de las personas encuestadas admitieron haber aumentado el uso de internet durante el confinamiento como medio de entretenimiento, y de estos el 11,2 \% demostraron un posible uso compulsivo de internet.

Los estudiantes universitarios son vulnerables a diferentes factores de riesgo como el uso indiscriminado de internet menguando la calidad del sueño. Por lo tanto, se propone 
realizar intervenciones en este grupo sobre la educación que es un factor importante para promover la salud del sueño y mejorar la calidad de vida.

\section{Conclusiones}

La necesidad de distanciamiento físico impuesta por la pandemia de COVID-19 en Ecuador, se ha visto acompañada por la existencia de una relación moderada entre el uso problemático de internet y la calidad de sueño. Es decir, el aumento del uso de internet coincide con la disminución de la calidad de sueño. Cabe recalcar que esta asociación no implica causalidad.

En los hallazgos reportados en la presente investigación se pudo corroborar que los estudiantes universitarios tienden a presentar una baja calidad de sueño la cual necesita tratamiento o atención médica. El aumento del uso problemático de internet puede ser causado por la nueva modalidad de estudio y trabajo que se ha tomado por la pandemia por COVID-19, en donde los universitarios pasan la mayor parte de tiempo usando aparatos electrónicos para continuar con sus estudios.

La finalidad del estudio, además de verificar la relación entre las variables descritas fue sentar un precedente de prevención en la salud de la población universitaria, puesto que está comprobado que la mala calidad de sueño puede generar problemas tanto físicos como psicológicos graves que pueden afectar el estilo de vida y siendo el uso de internet una actividad diaria sería recomendable regular su uso.

Asimismo, la facilidad de encontrar información actualizada de forma eficiente y segura a través del internet ha generado que la mayoría de personas abandonen el uso de libros físicos, generando otra posible razón por la que ha aumentado el uso de internet, además por el confinamiento generado por la pandemia actual es difícil que los universitarios se acerquen a las bibliotecas como lo hacían antes, sin embargo cabe recalcar que el uso problemático de internet se evidencia más en el tema de entretenimiento como el acceso a redes sociales, juegos o plataformas digitales.

En este aspecto, se sugiere advertir a esta población sobre las consecuencias de un uso inadecuado del internet para que lo utilicen de manera responsable y cuando sea posible promover el uso de libros físicos sugiriendo en las tareas que una cantidad de bibliografías sean provenientes de los mismos, así como también motivar a la lectura como una forma de entretenimiento.

Para finalizar, se recomienda evitar computadoras, teléfonos inteligentes y otros dispositivos en los lapsos previos a la hora de dormir. El factor más importante es ayudar a las personas a formar una comprensión razonable de la adicción a internet y cambiar estilos de vida poco saludables.

\section{Referencias}

[1]D. J. Kuss y O. López-Fernández, "Internet addiction and problematic Internet use: A systematic review of clinical research," World journal of psychiatry vol. 6, n 1, pp. 143-76, Marzo 2016.
[2]M. Vázquez-Chacón, S. Cabrejos-Llontop, Y. Yrigoin-Pérez, R. Robles-Alfaro y C. Toro-Huamanchumo, "Adicción a internet y calidad de sueño en estudiantes de medicina de una Universidad peruana, 2016," Revista Habanera de Ciencias Médicas, vol. 18, n 5, pp. 817-830, Octubre 2019.

[3]P. Carrillo-Mora, K. Barajas-Martínez, I. Sánchez-Vázquez y M. Rangel-Caballero, "Trastornos del sueño: ¿qué son y cuáles son sus consecuencias?," Revista de la Facultad de Medicina de la UNAM, vol. 61, $\mathrm{n}^{\circ}$ 1, pp. 6-20, Enero-febrero 2018.

[4]S. J. Kim, J. W. Kim, Y. S. Cho, K. J. Chung, H. Yoon y K. H. Kim, "Influence of Circadian Disruption Associated With Artificial Light at Night on Micturition Patterns in Shift Workers," International neurourology journal, vol. 23, $\mathrm{n}^{\circ} 4$, pp. 258-264, Diciembre 2019.

[5]J. Arendt y B. Middleton, "Human seasonal and circadian studies in Antarctica (Halley, $75^{\circ} \mathrm{S}$ )," General and Comparative Endocrinology, vol. 258, n 1, pp. 250-258, Marzo 2018. [6]P. Botero, P. Camargo, Y. Riaño y N. Velásquez, "Calidad del sueño en adultos según el grado de exposición a campos electromagnéticos en Bogotá y Duitama en 2017," Tesis de pregrado, Universidad de Ciencias Aplicadas y Ambientales, Bogotá, COL., 2017.

[7]H. Y. Wong, H. Y. Mo, M. N. Potenza, M. Chan, W. M. Lau, T. K. Chui, A. H. Pakpour y C. Y. Lin, "Relationships between Severity of Internet Gaming Disorder, Severity of Problematic Social Media Use, Sleep Quality and Psychological Distress," International journal of environmental research and public health, vol. 17, n 6, 1879, Marzo 2020.

[8]V. Parra, J. Vargas, B. Zamorano, F. Peña, Y. Velázquez, L. Ruiz y O. Monreal, "Adicción y factores determinantes en el uso problemático del Internet, en una muestra de jóvenes universitarios," Edutec-e, n 56, p. a337, Junio 2016.

[9]M. Laguna, "Estudio sobre el uso de internet y sus aplicaciones en el alumnado de último año de carrera de la Universidad de Alicante," Tesis Doctoral, Universidad de Alicante, Alicante, ESP., 2013.

[10]A. Rial, P. Gómez, M. Isorna, M. Araujo y J. Varela, "EUPI-a: Escala de Uso Problemático de Internet en adolescentes. Desarrollo y validación psicométrica," Adicciones, vol. 27, $\mathrm{n}^{\circ}$ 1, pp. 47-63, 2015.

[11]T. Fernández-Villa, J. Alguacil, A. Almaraz, J. Cancela, M. Delgado-Rodríguez, M. García-Martín, E. Jiménez-Mejías, J. Llorca, A. Molina, R. Ortíz, L. Félix y V. Martín, "Uso problemático de internet en estudiantes universitarios: factores asociados y diferencias de género," Adicciones, vol. 27, $\mathrm{n}^{\circ} 4$, pp. 265-275, 2015.

[12]S. Cañón, J. Castaño, D. Hoyos, J. Jaramillo, D. Leal, R. Rincón, E. Sánchez y L. Urueña, "Uso problemático de internet en estudiantes universitarios: factores asociados y diferencias de género," Adicciones, vol. 27, n 4, pp. 265-275, Julio-diciembre 2016.

[13]Z. Granados-Carrasco, A. Bartra-Aguinaga, D. Bendezú-Barnuevo, J. Huamanchumo-Merino, E. Hurtado-Noblecilla, J. Jiménez-Flores, F. León-Jiménez y D. Chang-Dávila, "Calidad del sueño en una facultad de medicina de 
Lambayeque," Anales de la Facultad de Medicina, vol. 74, $\mathrm{n}^{\circ}$ 4, pp. 311-314, Octubre-diciembre 2013.

[14]Q. Wang, K. Mati y Y. Cai, "The link between problematic internet use, problematic gaming, and psychological distress: does sleep quality matter?," BMC psychiatry, vol. 21, $\mathrm{n}^{\circ} 1,103$, Febrero 2021.

[15]M. Bautista y I. Taipe, "Calidad de sueño y factores asociados en internos rotativos de la carrera de Medicina de la Universidad de Cuenca 2019," Tesis de pregrado Médico Cirujano, Universidad de Cuenca, Cuenca, ECU., 2019.

[16]El Universo. (2020, Junio 26). El tráfico de internet en los hogares creció hasta $63 \%$ en medio de la pandemia del COVID-19. Diario El Universo [Internet]. Disponible en: https:// www.eluniverso.com/noticias/2020/06/23/nota/7881924/ internet-fijo-servicio-operadoras-demanda-cuarentena-covid-19

[17]B. Fernandes, U. Nanda, R. Tan-Mansukhani, A. Vallejo y C. Essau, "The impact of COVID-19 lockdown on internet use and escapism in adolescents," Revista de Psicología Clínica con Niños y Adolescentes, vol. 7, n 3, pp. 59-65, Septiembre 2020 .

[18]J. A. Casas, R. Ruiz-Olivares y R. Ortega-Ruiz, "Validation of the Internet and Social Networking Experiences Questionnaire in Spanish adolescents," International Journal of Clinical and Health Psychology, vol. 13, $n^{\circ}$ 1, pp. 40-48, Enero 2013.

[19]Y. Tan, Y. Chen, Y. Lu y L. Li, "Exploring Associations between Problematic Internet Use, Depressive Symptoms and Sleep Disturbance among Southern Chinese Adolescents," International journal of environmental research and public health, vol. 13, $\mathrm{n}^{\circ}$ 3, 313, Marzo 2016.

[20]A. Barquero y F. Calderón, "Influencia de las nuevas tecnologías en el desarrollo adolescente y posibles desajustes," Revista Cúpula, vol. 30, n 2, pp. 11-25, 2016.

[21]J. P. C. Chang y C. C. Hung. Uso problemático de internet. Libro electrónico de IACAPAP de Salud Mental en Niños y Adolescentes. Geneva: Asociación Internacional de Psiquiatría y Profesiones Aliadas de Niños y Adolescentes. [Internet]. 2016. Disponible en: https://iacapap.org/content/ uploads/H.6-Uso-problem\%C3\%A1 tico-de-Internet-SPANISH-2017.pdf

[22]S. García y G. Zurita, "Estudio estadístico de internet y su incidencia en la educación universitaria fiscal de la provincia del Guayas," Tesis de pregrado Ingeniera en Estadística
Informática, Escuela Superior Politécnica del Litoral (ESPOL), Guayaquil, ECU., 2017.

[23]J. Redondo, K. Rangel, M. Luzardo y C. Inglés, “Experiencias relacionadas con el uso de internet y celular en una muestra de estudiantes universitarios colombianos," Revista Virtual Universidad Católica del Norte, vol. 49, pp. 7-22, Septiembre-diciembre 2016.

[24]S. Cruces, R. Guil, N. Sánchez y J. Pereira, "Consumo de nuevas tecnologías y factores de personalidad en estudiantes universitarios," Commons, vol. 5, n 2, pp. 203-228, Diciembre 2016.

[25]X. Carbonell, A. Chamarro, M. Griffiths, U. Oberst, R. Cladellas y A. Talam, "Problematic Internet and cell phone use in Spanish teenagers and young students," Anales de psicología, vol. 8, n³, pp. 789-796, Octubre 2012.

[26]S. Iñiguez-Jiménez, S. Cruz-Pierard y S. Vaca-Córdova, "Uso problemático de tecnologías de la información y comunicación, consumo de sustancias y su impacto en la salud mental de estudiantes de bachillerato," PAIDEIA XXI, vol. 10, $\mathrm{n}^{\circ} 2$, pp. 471-489, Julio-diciembre 2020.

[27]M. Machado-Duque, J. Echeverri y J. Machado-Alba, "Somnolencia diurna excesiva, mala calidad del sueño y bajo rendimiento académico en estudiantes de Medicina," Revista Colombiana de Psiquiatría, vol. 44, n 3, pp. 137-142, Julio-septiembre 2015.

[28]S. de la Portilla, C. Dussán, D. Montoya, J. Taborda y L. Nieto, "Calidad de sueño y somnolencia diurna excesiva en estudiantes universitarios de diferentes dominios," Hacia Promoc. Salud, vol. 24, n 1, pp. 84-96, Enero - junio 2019. [29]F. Wang y É. Bíró, “Determinants of sleep quality in college students: A literature review," Explore, vol. 17, $\mathrm{n}^{\circ} 2, \mathrm{pp}$. 170-177, Marzo-abril 2021.

[30]M. Puerto, D. Rivero, L. Sansores, L. Gamboa y L. Sarabia, "Somnolencia, hábitos de sueño y uso de redes sociales en estudiantes universitarios," Enseñanza e Investigación en Psicología, vol. 20, n 2, pp. 189-195, Mayo-agosto 2015.

[31]Ministerio de Sanidad. "COVID-19, consumo de sustancias psicoactivas y adicción”. Observatorio Español de las Drogas y las Adicciones, España. Informe Impacto COVID. [Internet]. Julio 2020. Disponible en: https://pnsd.sanidad.gob.es/noticiasEventos/actualidad/2020_Coronavirus/ pdf/20200715_Informe_IMPACTO_COVID-19_OEDA_final.pdf 